\title{
Metal Stent Insertion for Malignant Obstruction of a Colostomy
}

\author{
Antonios Wehbeh ${ }^{1}$, Mahmoud Rahal ${ }^{2}$, Hala Fatima ${ }^{1}$ \\ 1. Gastroenterology and Hepatology, Indiana University School of Medicine, Indianapolis, USA 2. Internal Medicine, \\ Indiana University School of Medicine, Indianapolis, USA
}

Corresponding author: Antonios Wehbeh,wehbehantonios@gmail.com

\begin{abstract}
A 47-year-old female with metastatic cervical cancer and diverting colostomy presented with abdominal distention and minimal stool output from her colostomy. A computed tomography (CT) scan revealed a metastatic mass causing partial obstruction at the colostomy level and significant proximal colonic dilation. Her obstruction was relieved by the endoscopic placement of a metal stent through the stoma, with the stent's distal edge visible externally but not protruding beyond skin level. Two months later, the stent remained patent and did not migrate. This case highlights a viable palliative treatment option for patients
\end{abstract} who are not operative candidates.

Categories: Gastroenterology, General Surgery

Keywords: colostomy, colonic obstruction, metal stent, colonoscopy

\section{Introduction}

Malignant obstruction of colostomy is a rare occurrence that is traditionally treated with surgical intervention. However, when surgery is not a feasible option, the recommendations for its management are unclear [1]. Self-expandable metallic stents (SEMS) have been used for the relief of malignant colonic obstruction [2], yet only a few reports describing the use of stents for colostomy obstruction are available [1, $3,4]$. In this article, we report a case where a colonic stent was successfully placed to relieve malignant stomal obstruction due to metastatic cervical cancer.

Received 06/26/2020 Review began 07/05/2020 Review ended 08/22/2020 Published 08/29/2020

(c) Copyright 2020 Wehbeh et al. This is an open access article distributed under the terms of the Creative Commons Attribution License CC-BY 4.0., which permits unrestricted use, distribution, and reproduction in any medium, provided the original author and source are credited.
This case report was presented as a poster at the 2019 American College of Gastroenterology (ACG) Annual Meeting, San Antonio, Texas, and it was published as an accepted meeting presentation in The American Journal of Gastroenterology, October 2019, supplement issue.

\section{Case Presentation}

A 47-year-old female with a past history of hypertension and metastatic cervical cancer presented with abdominal distention and minimal stool output from her ostomy for two weeks duration. She described having small amounts of liquid stools which gradually decreased to the point of only mucous discharge from her ostomy. She had anorexia, abdominal cramping, and bloating with food intake, but no nausea or vomiting. Three years prior, she was diagnosed with stage IIB cervical cancer after presenting with abnormal vaginal bleeding. Her disease progressed rapidly despite chemotherapy and radiation. It was complicated by rectovaginal fistula for which she underwent laparoscopic diverting colostomy years prior to presentation. The patient had recently noted an enlarging mass near the ostomy site which was biopsied and showed metastatic adenocarcinoma of endocervical origin.

On presentation, examination revealed a soft but distended abdomen with palpable peristomal subcutaneous masses. A CT scan of the abdomen and pelvis showed a large heterogeneous mass adjacent to the colostomy with both intra-abdominal and extra-abdominal components. The mass was causing compression and partial obstruction at the level of the colostomy with significant proximal colonic dilation measuring up to $8 \mathrm{~cm}$ in the cecum and ascending colon (Figure 1). 


\section{Cureus}

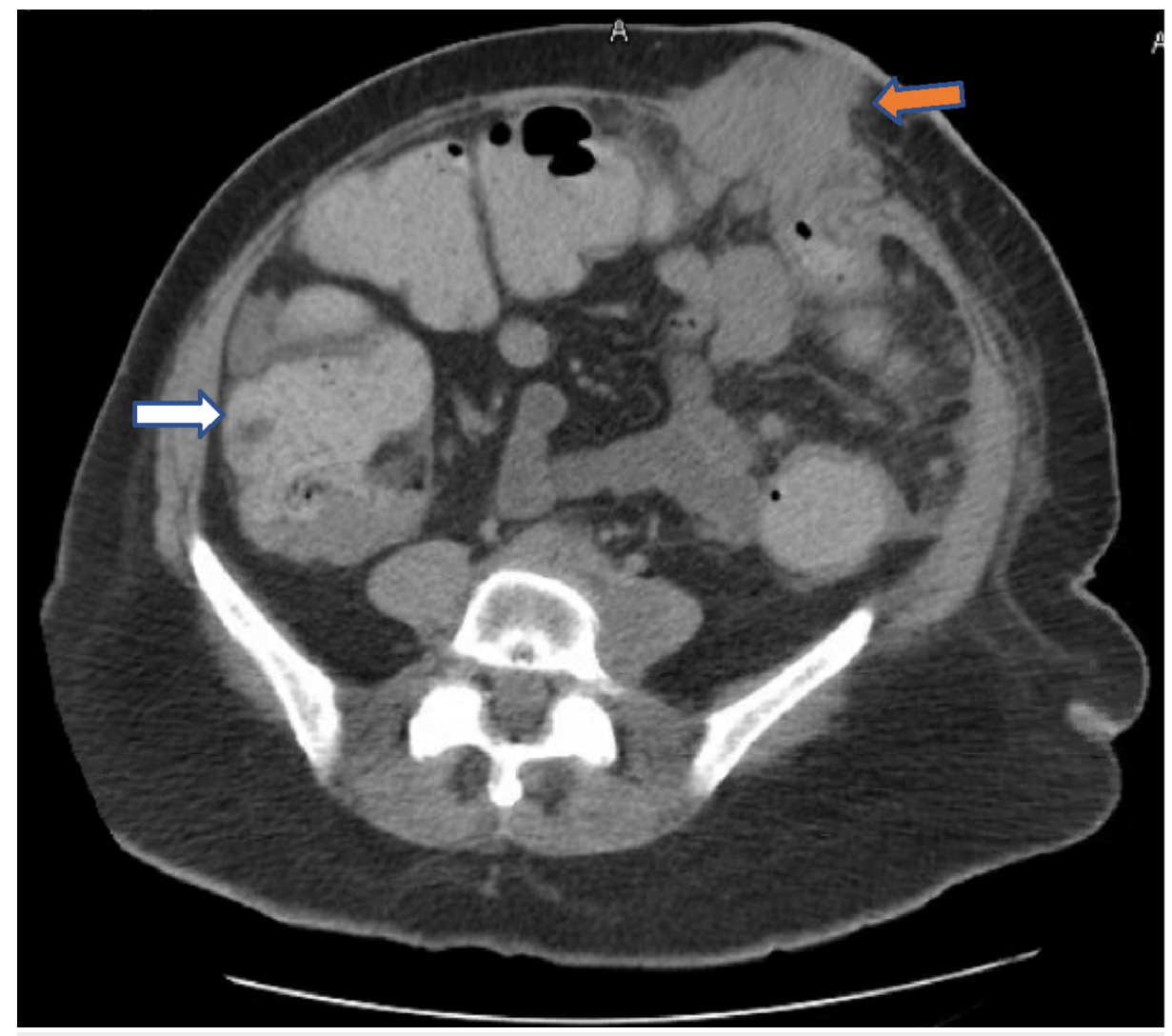

FIGURE 1: Axial cuts of an abdominal CT scan showing obstruction at the level of the colostomy by a mass (orange arrow), resulting in significant proximal colonic dilation (white arrow)

As a temporizing measure, a red rubber catheter was passed through the stoma to irrigate and decompress the colon. Given the extensive metastatic disease, she was not a candidate for any operative intervention, and subsequently, endoscopic stent placement was pursued.

Colonoscopy was performed under moderate sedation. Examination showed a severe stenosis $4 \mathrm{~cm}$ in length at the surgical stoma which was traversed with the adult colonoscope. A $25 \mathrm{~mm}$ x $6 \mathrm{~cm}$ covered selfexpandable metal stent (SEMS) was successfully placed with the distal edge visible externally but not protruding beyond skin level (Figures $2 a, 2 b$ ).
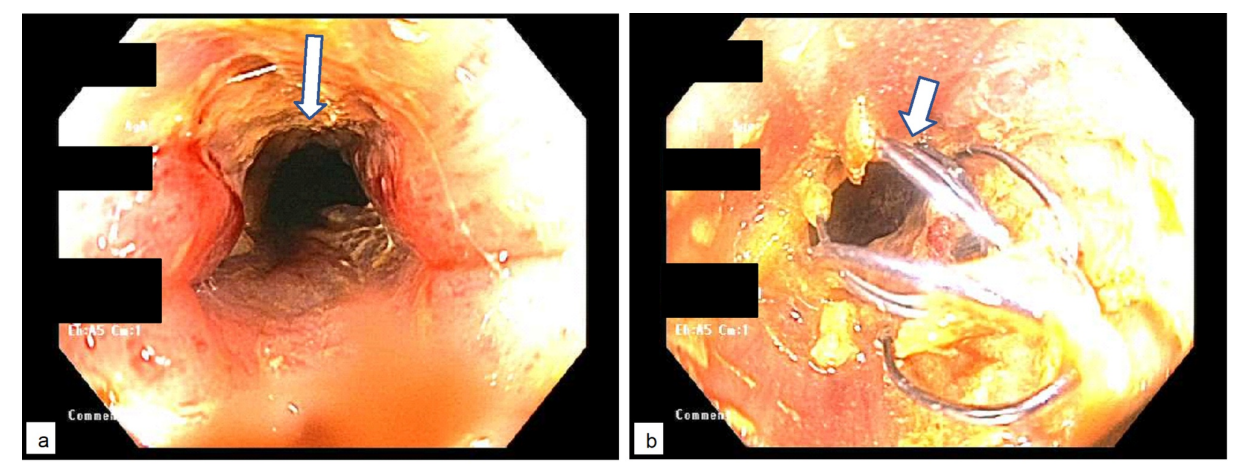

FIGURE 2: Colonoscopy showing severe stenosis at the stoma (a), treated with placement of a covered metal stent (b)

No complications occurred during or after the procedure. After the placement of the stent, her abdominal discomfort resolved. She started to have good stool output and tolerated a regular diet. 


\section{Cureus}

She presented two months later with draining fistula tracts around the ostomy, and a CT scan showed a patent stent still in place without recurrent colonic obstruction (Figures 3a, 3b).
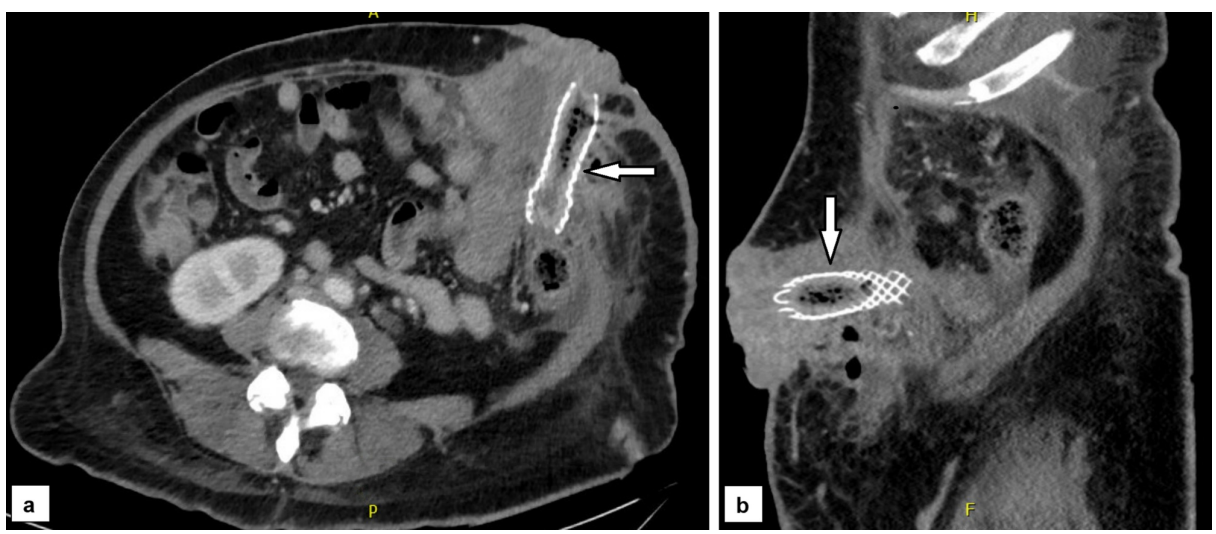

FIGURE 3: Abdominal CT scan showing the metal stent in place, as seen on axial (a) and sagittal cuts (b)

\section{Discussion}

Stoma construction is a common procedure performed in the setting of colon cancer, trauma, diverticulitis, ischemic colitis, inflammatory bowel disease, and fistulas [5]. Stenosis and malignant colostomy obstruction are among the complications of stoma construction $[6,7]$. Surgery is usually the traditional treatment of these complications, however, there is limited data available regarding the management of patients who are poor surgical candidates.

SEMS can be used both as a bridge to surgery or as a palliative measure in patients with a malignant colonic obstruction due to metastatic disease and unresectable colon cancer tumor. There is no data on whether SEMS has lower morbidity or mortality when compared to palliative surgery in this group of patients. There are a few case reports describing the use of either covered or uncovered SEMS for colostomy obstruction, all of which remained patent until the patients' death 1.5 - 6 months later $[1,3,4]$.

\section{Conclusions}

In conclusion, metal stent insertion for malignant obstruction of colostomy could be a viable palliative treatment option in patients who are not operative candidates. Further studies are needed to assess the effectiveness and safety of metal stents in improving obstructive symptoms compared with palliative surgery.

\section{Additional Information}

\section{Disclosures}

Human subjects: Consent was obtained by all participants in this study. Conflicts of interest: In compliance with the ICMJE uniform disclosure form, all authors declare the following: Payment/services info: All authors have declared that no financial support was received from any organization for the submitted work. Financial relationships: All authors have declared that they have no financial relationships at present or within the previous three years with any organizations that might have an interest in the submitted work. Other relationships: All authors have declared that there are no other relationships or activities that could appear to have influenced the submitted work.

\section{References}

1. Wi JO, Shin SJ, Yoo JH, et al.: Insertion of self expandable metal stent for malignant stomal obstruction in a patient with advanced colon cancer. Clin Endosc. 2012, 45:448-50. 10.5946/ce.2012.45.4.448

2. Arezzo A, Passera R, Secco GL, et al.: Stent as bridge to surgery for left-sided malignant colonic obstruction reduces adverse events and stoma rate compared with emergency surgery: results of a systematic review and meta-analysis of randomized controlled trials. Gastrointest Endosc. 2017, 86:416-426. 10.1016/.gie.2017.03.1542

3. Kim WH, Kwon CI, Kim JW, Lee C: Insertion of a self-expanding metal stent for a stomal stenosis . Endoscopy. 2012, 2:143-4. 10.1055/s-0032-1306904

4. Gils NAV, Cornelissen JGHM, Tan A: A metal stent in a colostomy obstruction . GE Port J Gastroenterol. 2018, 25:214-215. 10.1159/000481625

5. Duchesne JC, Wang YZ, Weintraub SL, Boyle M, Hunt JP: Stoma complications: A multivariate analysis . Am Surg. 2002, 11:961-6. 


\section{Cureus}

6. Shellito, P.C: Complications of abdominal stoma surgery. Dis Colon Rectum. 1998, 41:1562-72. 10.1007/BF02237308

7. Shabbir J, Britton DC: Stoma complications: a literature overview . Colorectal Dis. 2010, 12:958-64. 10.1111/j.1463-1318.2009.02006.x 